\title{
Correction to: Redox Status and Antioxidative Cofactor Metals Influence Clinical and Pathological Characteristics of Papillary Thyroid Carcinoma and Colloid Goiter
}

\author{
Branislav Rovcanin ${ }^{1}$ - Aleksandar Stojsavljevic ${ }^{2}$ - Dusan Kekic ${ }^{3} \cdot$ Kristina Gopcevic $^{4}$ - Dragan Manojlovic ${ }^{2,5}$. \\ Milan Jovanovic ${ }^{1}$ - Sasa Knezevic ${ }^{6}$. Vladan Zivaljevic ${ }^{1}$ - Aleksandar Diklic ${ }^{1} \cdot$ Ivan Paunovic $^{1}$
}

Published online: 18 April 2020

(C) Springer Science+Business Media, LLC, part of Springer Nature 2020

\section{Correction to: Biological Trace Element Research} https://doi.org/10.1007/s12011-019-01995-x

The original version of this article unfortunately contained a mistake. The author names in the author group are now presented correctly.

The original article has been updated.

The online version of the original article can be found at https://doi.org/ 10.1007/s12011-019-01995-x

\section{Branislav Rovcanin}

rovcaninb@yahoo.com

1 Center for Endocrine Surgery, Clinical Center of Serbia, Faculty of Medicine, University of Belgrade, Belgrade, Serbia

2 Faculty of Chemistry, University of Belgrade, Studentski trg 12-16, Belgrade, Serbia

3 Institute for Microbiology and Immunology, Faculty of Medicine, University of Belgrade, Belgrade, Serbia

4 Institute for Chemistry in Medicine, Faculty of Medicine, University of Belgrade, Belgrade, Serbia

5 South Ural State University, Lenin Prospect 76, Chelyabinsk, Russia

6 Center for Anesthesiology and Resuscitation, Clinical Center of Serbia, Belgrade, Serbia 